\title{
Numerical solution of telegraph equation by using LT inversion technique
}

\author{
Mohammad Javidi*, Nemat Nyamoradi \\ Department of Mathematics, Faculty of science, Razi University, Kermanshah, 67149, Iran \\ ${ }^{*}$ Corresponding author E-mail: Mo_javidi@yahoo.com
}

\begin{abstract}
In this paper, the numerical solution of one-dimensional linear hyperbolic telegraph equation, which describe wave propagation of electric signals in a cable transmission line, is proposed employing the well known homotopy perturbation method(HPM) and laplace transform(LT). Using Laplace transform scheme, the problem is converted into a partial differential equation without any differentiation respect to time. Homotopy perturbation method is applied in the Laplace transform domain. We use Stehfest's numerical inversion algorithm of Laplace transform to retrieve the time domain solution. The performance was found to be very good. The approximate solutions are in excellent agreement with those by the Chebyshev spectral collocation method (CSCM) and the method uses interpolating scaling functions. To illustrate the method some examples are provided. The results show the simplicity and the efficiency of the method.
\end{abstract}

Keywords: Laplace transform; homotopy perturbation method; telegraph equation; Stehfest's numerical inversion algorithm.

\section{Introduction}

Consider one dimensional linear hyperbolic telegraph equation

$$
\frac{\partial^{2} u}{\partial t^{2}}+2 \alpha \frac{\partial u}{\partial t}+\beta^{2} u=\frac{\partial^{2} u}{\partial x^{2}}+f(x, t), \quad(x, t) \in[0,1] \times[0, T] .
$$

with initial conditions

$$
u(x, 0)=f_{0}(x), \quad \frac{\partial u}{\partial t}(x, 0)=f_{1}(x),
$$

and boundary conditions

$$
u(0, t)=g_{0}(x), \quad u(1, t)=g_{1}(x), \quad t \geq 0 .
$$

Telegraph equation is commonly used in the study of wave propagation of electric signals in a cable transmission line and also in wave phenomena. Many researchers have used various numerical and analytical methods to solve the telegraph equation. Mohebbi and Dehaghan [1], studied high order compact solution to solve the telegraph equation. Gao and Chi [2], used unconditionally stable difference scheme for a one-space dimensional linear hyperbolic equation. Saadatmandi and Dehghan [3], developed a numerical solution based on Chebyshev Tau method. Yousefi [4], used Legendre multi wavelet Galerkin method for solving the hyperbolic telegraph equation. Dehghan and Ghesmati [5], developed a numerical approach based on the truly meshless local weakstrong (MLWS) methods to deal with the second order two-space-dimensional telegraph equation. To solve the telegraph equation using the MLWS method, the conventional moving least squares (MLS) approximation is exploited in order to interpolate the solution of the equation. A time stepping scheme is employed to approximate the time derivative. Das and Gupta [6], used homotopy analysis method for solving fractional hyperbolic partial differential equations. By using initial values, the explicit solutions of telegraph equation for different particular cases have been derived. Abdou [7], 
used Adomian decomposition method for solving the telegraph equation in charged particle transport. Lakestani and Saray [8], developed a numerical technique for the solution of second order one dimensional linear hyperbolic equation. The method consists of expanding the required approximate solution as the elements of interpolating scaling functions. In their technique, by using the operational matrix of derivatives, they reduced the problem to a set of algebraic equations. Mohanty [9], made investigations on the one-space-dimensional hyperbolic equations. Mohanty [10], carried over a new technique to solve the linear one-space-dimensional hyperbolic Eq. (1), which is unconditionally stable and is of second-order accuracy in both the time and space components. Also Mohanty [10], proposed a three level implicit unconditionally stable difference scheme proposed by Lapidus and Pinder [11], of second-order accuracy in both time and space variables for the solution of (1) with variable coefficients such that fictitious points are not needed at each time step along the boundary. Homotopy analysis method is developed by Hashim et al. [12], to solve fractional IVPs. Borhanifar and Abazari [13], developed an unconditionally stable parallel difference scheme for telegraph equation. A numerical scheme is developed by Borhanifar and Abazari [13], to solve the one-dimensional hyperbolic telegraph equation using collocation points Dehghan and Shokri [14], and approximating the solution using a thin plate splines radial basis function. Another numerical method is presented by Dehghan and Lakestani [15], to solve the one-dimensional hyperbolic telegraph equation using Chebyshev cardinal functions. Also several test problems are given and the results of numerical experiments are compared with analytical solutions to confirm the good accuracy of the presented scheme. Differential transform method (DTM) is considered by Biazar and Eslami [16], to solve telegraph equation. Using differential transform method, it is possible to find the exact solution or a closed approximate solution of an equation.

In this paper we use Laplace transform homotopy perturbation method to solve Eq. (1) with the initial and boundary conditions (2-3). The outline of this paper is as follows. Section 1 contains a brief summary on telegraph equation. In Section 2, we review some of the standard facts on HPM. In the third section, we develop the theory of Lt-HPM. In section 4, the numerical results of applying the method of this article on some test problems for the Eq. (1) are presented.

\section{Homotopy perturbation method}

Recently various powerful mathematical methods such as parameter-expansion method by Xu [17], variational iteration method by He [18], Exp-function method by Javidi and Golbabai [19], F-expansion method by He and Abdou [20], spectral domian decomposition approach by Golbabai and Javidi [21], and Nonlinear relaxation phenomena by Draganescu and Capalnasan [22], have been proposed to obtain exact and approximate analytic solutions for linear and nonlinear problems. The application of homotopy perturbation method in linear and nonlinear problems has been devoted by scientists and engineers, because this method is to continuously deform a simple problem which is easy to solve into the under study problem which is difficult to solve. This method was proposed first by He [23], and systematical description in 2000 which is, in fact, a coupling of the traditional perturbation method and homotopy in topology. This method was further developed and improved by He and applied to non-linear oscillators with discontinuities by He [24], nth order integro-differential equations by Golbabai and M. Javidi [25], asymptotology by $\mathrm{He}$ [26], boundary value problem by He [27], Limit cycle and bifurcation of nonlinear problems by He [28] and many other subjects. Thus He's method is a universal one which can solve various kinds of nonlinear equations. To explain HPM, we consider a general equation of the form

$$
L(u)=0
$$

where $L$ is an integral or differential operator. For solving Eq. (1), we may choose a convex homotopy defined by

$$
H(u, p)=(1-p) F(u)+p L(u)=0,
$$

where $F$ is an operator that we can solve the equation $F(u)=0$ easily. From (5), we have

$$
H(u, 0)=F(u), \quad H(u, 1)=L(u)=0,
$$

Therefore the convex homotopy (5) continuously trace an implicitly defined curve from a starting point $H(u, 0)=0$ to a solution function $H(u, 1)=0$. The embedding parameter $p$ monotonically increases from zero to unit as trivial problem $F(u)=0$ is continuously deformed to original problem $L(u)=0$. The HPM uses the homotopy parameter $p$ as an expanding parameter to obtain the solution of(4)as:

$$
v=u_{0}+p u_{1}+p^{2} u_{2}+\cdots,
$$


when $p \rightarrow 1$, (6) becomes the solution of equation (4), i.e.,

$$
u=\lim _{p \rightarrow 1} v=u_{0}+u_{1}+u_{2}+\cdots .
$$

Substituting (6) into (5) results into

$$
(1-p) F\left(u_{0}+p u_{1}+p^{2} u_{2}+\cdots\right)+p L\left(u_{0}+p u_{1}+p^{2} u_{2}+\cdots\right)=0
$$

Equating the terms with identical powers of $p$, we can obtain $u_{j}, j=0,1,2, \cdots$.

\section{Laplace transform HPM}

Let us define the LT operator $\varphi$ on a function $u(x, t),(t \geq 0)$ by

$$
\varphi\{u(x, t) ; t \mapsto s\}=\int_{0}^{\infty} e^{-s t} u(x, t) d t
$$

and denote it by $\varphi\{u(x, t) ; t \mapsto s\}=L(u(x, s))$, where $s$ is the LT parameter. For our purpose here, we shall take $s$ to be real and positive. The inverse problem (9) is usually known as the real inversion problem.

Taking the Laplace transform of (1) we obtain a new partial differential equation as follows

$$
\left(s^{2}+2 \alpha s+\beta^{2}\right) v(x, s)=(s+2 \alpha) f_{0}(x)+f_{1}(x)+\frac{\partial^{2}}{\partial x^{2}} v(x, s)+F(x, s)
$$

where $v(x, s)$ and $F(x, s)$ are the Laplace transform of $u(x, t)$ and $f(x, t)$ respectively.

The Laplace transform of the boundary conditions in Eq. (3) is of the form:

$$
v(0, s)=L\left(g_{0}(t)\right), v(1, s)=L\left(g_{1}(t)\right),
$$

According to HPM, for solving Eq. (10) we construct a homotopy by Madani et al. [29], as the following form:

$$
\left(s^{2}+2 \alpha s+\beta^{2}\right) v(x, s)=(s+2 \alpha) f_{0}(x)+f_{1}(x)+p \frac{\partial^{2}}{\partial x^{2}} v(x, s)+F(x, s) .
$$

Now let us present the solution of Eq. (12) as the following form

$$
\bar{v}(x, s)=\sum_{j=0}^{\infty} p^{j} v_{j}(x, s)
$$

where $v_{j}(x, s), j=0,1,2, \ldots$, are functions which should be determined. By substituting (13) into (12), we have

$$
\begin{aligned}
\left(s^{2}+2 \alpha s+\beta^{2}\right) \sum_{j=0}^{\infty} p^{j} v_{j}(x, s) & \\
& =(s+2 \alpha) f_{0}(x)+f_{1}(x)+p \frac{\partial^{2}}{\partial x^{2}} \sum_{j=0}^{\infty} p^{j} v_{j}(x, s)+F(x, s) .
\end{aligned}
$$

Equating the coefficients of $p$ with the same powers in (14) leads to

$$
\begin{aligned}
& p^{0}: v_{0}(x, s)\left(s^{2}+2 \alpha s+\beta^{2}\right)=(s+2 \alpha) f_{0}(x)+f_{1}(x)+F(x, s), \\
& p^{1}: v_{1}(x, s)\left(s^{2}+2 \alpha s+\beta^{2}\right)=\frac{\partial^{2}}{\partial x^{2}} v_{0}(x, s) \\
& p^{2}: v_{2}(x, s)\left(s^{2}+2 \alpha s+\beta^{2}\right)=\frac{\partial^{2}}{\partial x^{2}} v_{1}(x, s) \\
& \vdots \\
& p^{n+1}: v_{n+1}(x, s)\left(s^{2}+2 \alpha s+\beta^{2}\right)=\frac{\partial^{2}}{\partial x^{2}} v_{n}(x, s) .
\end{aligned}
$$


From (15), we have

$$
\begin{aligned}
& v_{0}(x, s)=\frac{(s+2 \alpha) f_{0}(x)+f_{1}(x)+F(x, s)}{s^{2}+2 \alpha s+\beta^{2}}, \\
& v_{1}(x, s)=\frac{1}{s^{2}+2 \alpha s+\beta^{2}} \frac{\partial^{2}}{\partial x^{2}} v_{0}(x, s), \\
& v_{2}(x, s)=\frac{1}{s^{2}+2 \alpha s+\beta^{2}} \frac{\partial^{2}}{\partial x^{2}} v_{1}(x, s), \\
& \vdots \\
& v_{n+1}(x, s)=\frac{1}{s^{2}+2 \alpha s+\beta^{2}} \frac{\partial^{2}}{\partial x^{2}} v_{n}(x, s) .
\end{aligned}
$$

when $p \rightarrow 1$, (13) becomes the approximate solution of equation (10), i.e.,

$$
v(x, s) \simeq H_{n}(x, s)=\sum_{j=0}^{n} v_{j}(x, s)
$$

Taking the inverse LT from both sides of (17) we have the approximate solution of (1)-(3).

$$
u(x, t) \simeq W_{n}(x, t)=L^{-1}\left(H_{n}(x, s)\right) .
$$

The physical solution $u(x, t)$ can be recovered approximately from $H_{n}(x, s)$ according to the Stehfest's [30], algorithm as follows

$$
u_{n}(x, t)=\frac{\ln (2)}{t} \sum_{j=1}^{2 p} d_{j} H_{n}\left(x, j \frac{\ln (2)}{t}\right) .
$$

were $p$ is positive integer and

$$
d_{j}=(-1)^{j+p} \sum_{i=\left[\frac{j+1}{2}\right]}^{\min (j, p)} \frac{i^{p}(2 i) !}{(p-i) ! i !(i-1) !(j-i) !(2 i-j) !} .
$$

where $[r]$ denotes the integer part of the real number $\mathrm{r}$.

\section{Numerical results}

In this section the LHPM applied on several test problems to show the efficiency and accuracy of the new method. In examples 1-2 we consider $p=7$.

Example1. In example 1 we consider the following telegraph equation by Lakestani and Nemati Saray [8].

$$
\frac{\partial^{2} u}{\partial t^{2}}+2 \alpha \frac{\partial u}{\partial t}+\beta^{2} u=\frac{\partial^{2} u}{\partial x^{2}}+\left(3-4 \alpha+\beta^{2}\right) e^{-2 t} \sinh (x),
$$

with initial conditions

$$
u(x, 0)=\sinh (x), \frac{\partial u}{\partial t}(x, 0)=-2 \sinh (x)
$$

and boundary conditions

$$
u(0, t)=0, u(1, t)=e^{-2 t} \sinh (1) .
$$

The exact solution is given by

$$
u(x, t)=e^{-2 t} \sinh (x) .
$$

Taking the Laplace transform of (19) we obtain a new partial differential equation as follows

$$
\left(s^{2}+2 \alpha s+\beta^{2}\right) v(x, s)=\frac{\partial^{2}}{\partial x^{2}} v(x, s)+\frac{1}{s+2}\left(1-\frac{1}{s^{2}+2 \alpha s+\beta^{2}}\right) \sinh (x),
$$


with the following boundary conditions

$$
v(0, s)=0, v(1, s)=\frac{\sinh (1)}{s+2} .
$$

By substitution of (13) in (23) and equating the coefficients of $p$ with the same powers leads to

$$
\begin{aligned}
& v_{0}(x, s)=\frac{1}{s+2}\left(1-\frac{1}{s^{2}+2 \alpha s+\beta^{2}}\right) \sinh (x) \\
& v_{1}(x, s)=\frac{1}{s+2}\left(\frac{1}{s^{2}+2 \alpha s+\beta^{2}}-\frac{1}{\left(s^{2}+2 \alpha s+\beta^{2}\right)^{2}}\right) \sinh (x) \\
& v_{2}(x, s)=\frac{1}{s+2}\left(\frac{1}{\left(s^{2}+2 \alpha s+\beta^{2}\right)^{2}}-\frac{1}{\left(s^{2}+2 \alpha s+\beta^{2}\right)^{3}}\right) \sinh (x) \\
& \vdots \\
& v_{n}(x, s)=\frac{1}{s+2}\left(\frac{1}{\left(s^{2}+2 \alpha s+\beta^{2}\right)^{n}}-\frac{1}{\left(s^{2}+2 \alpha s+\beta^{2}\right)^{n+1}}\right) \sinh (x) .
\end{aligned}
$$

Thus we can evaluate the approximate solution of Eq. (23) as follows

$$
H_{n}(x, s)=\sum_{j=0}^{n} v_{j}(x, s)=\frac{1}{s+2}\left(1-\frac{1}{\left(s^{2}+2 \alpha s+\beta^{2}\right)^{n+1}}\right) \sinh (x) .
$$

Now from Stehfest formula and Eq. (26) we have

$$
u_{n}(x, t)=\frac{\ln (2)}{t} \sum_{j=1}^{2 p} d_{j} \frac{1}{j \frac{\ln (2)}{t}+2}\left(1-\frac{1}{\left(\left(j \frac{\ln (2)}{t}\right)^{2}+2 \alpha\left(j \frac{\ln (2)}{t}\right)+\beta^{2}\right)^{n+1}}\right) \sinh (x) .
$$

Table 1 show the absolute error $\left|u(1, t)-u_{n}(1, t)\right|$ using the LHPM with $\alpha=20, \beta=10$ for various values of $n$ and $t$. As can be seen from the table, the numerical solutions are in good agreement with the exact solution. In Table 2, we list the results obtained by LT-HPM method and compared with the results given in Lakestani and Nemati Saray [8], with $\beta=10, \alpha=20$ for various values of $x$ and $t$. As we seen from this table, it is clear that the results obtained by LT-HPM is superior to that obtained by Lakestani and Nemati Saray [8].

Example 2. Consider the following telegraph equation

$$
\frac{\partial^{2} u}{\partial t^{2}}+2 \alpha \frac{\partial u}{\partial t}+\beta^{2} u=\frac{\partial^{2} u}{\partial x^{2}}-2 \alpha \sin (t) \sin (x)+\beta^{2} \cos (t) \sin (x),
$$

with initial conditions

$$
u(x, 0)=\sin (x), \frac{\partial u}{\partial t}(x, 0)=0,
$$

and boundary conditions

$$
u(0, t)=0, u(1, t)=\cos (t) \sin (1) .
$$

The exact solution is given by

$$
u(x, t)=\cos (t) \sin (x) .
$$

Taking the Laplace transform of (28) we obtain a new partial differential equation as follows

$$
\left(s^{2}+2 \alpha s+\beta^{2}\right) v(x, s)=\frac{\partial^{2}}{\partial x^{2}} v(x, s)+\frac{s\left(s^{2}+2 \alpha s+\beta^{2}\right)}{s^{2}+1} \sin (x)
$$

with the following boundary conditions

$$
v(0, s)=0, v(1, s)=\frac{\sin (1)}{s^{2}+1} .
$$

By substitution of (13) in (32) and equating the coefficients of $p$ with the same powers leads to

$$
\begin{aligned}
& v_{0}(x, s)=\frac{s}{s^{2}+1}\left(1-\frac{1}{s^{2}+2 \alpha s+\beta^{2}}\right) \sin (x), \\
& v_{1}(x, s)=\frac{s}{s^{2}+1}\left(\frac{1}{s^{2}+2 \alpha s+\beta^{2}}-\frac{1}{\left(s^{2}+2 \alpha s+\beta^{2}\right)^{2}}\right) \sin (x), \\
& v_{2}(x, s)=\frac{s}{s^{2}+1}\left(\frac{1}{\left(s^{2}+2 \alpha s+\beta^{2}\right)^{2}}-\frac{1}{\left(s^{2}+2 \alpha s+\beta^{2}\right)^{3}}\right) \sin (x), \\
& \vdots \\
& v_{n}(x, s)=\frac{s}{s^{2}+1}\left(\frac{1}{\left(s^{2}+2 \alpha s+\beta^{2}\right)^{n}}-\frac{1}{\left(s^{2}+2 \alpha s+\beta^{2}\right)^{n+1}}\right) \sin (x) .
\end{aligned}
$$




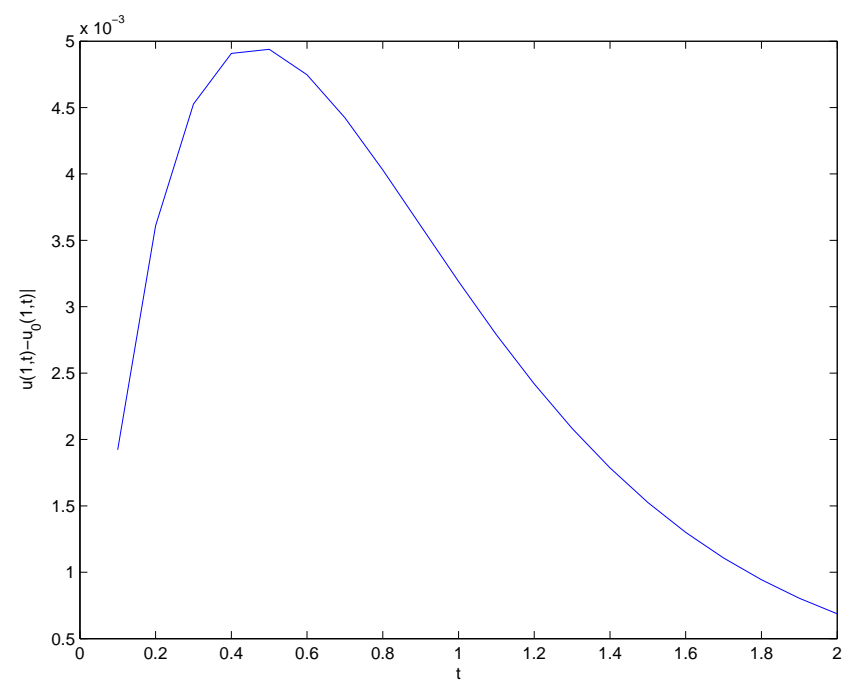

Figure 1: Absolute error $\left|u(1, t)-u_{0}(1, t)\right|$ for $\alpha=20, \beta=10$ for example 1 .

Thus we can evaluate the approximation of the solution of Eq. (32) as follows

$$
v(x, s) \simeq H_{n}=\sum_{j=0}^{n} v_{j}(x, s)=\frac{s}{s^{2}+1}\left(1-\frac{1}{\left(s^{2}+2 \alpha s+\beta^{2}\right)^{n+1}}\right) \sin (x) .
$$

From Stehfest formula and Eq. (35) we have

$$
u_{n}(x, t)=\frac{\ln (2)}{t} \sum_{j=1}^{2 p} d_{j} \frac{j \frac{\ln (2)}{t}}{\left(j \frac{\ln (2)}{t}\right)^{2}+1}\left(1-\frac{1}{\left(\left(j \frac{\ln (2)}{t}\right)^{2}+2 \alpha\left(j \frac{\ln (2)}{t}\right)+\beta^{2}\right)^{n+1}}\right) \sin (x) .
$$

Table 3 show the absolute error $\left|u(1, t)-u_{n}(1, t)\right|$ using the technique presented in the previous section with $\alpha=20, \beta=10$ for various values of $n$ and $t$.

In Table 4, we list the results obtained by LT-HPM and compared with CSCM results given in Javidi [31], for $\beta=10, \alpha=20$ for various values of $x$ and $t$. As we seen from this table, it is clear that the results obtained by LT-HPM is superior to that obtained by CSCM. In Figure 1, we plot absolute error $\left|u(1, t)-u_{0}(1, t)\right|$ at $x=1$ with $\alpha=20, \beta=10$. In Figure 2, we plot absolute error $\left|u(1, t)-u_{0}(1, t)\right|$ at $x=1$ with $\alpha=20, \beta=10$. In Figure 3, we plot absolute error $\left|u(1, t)-u_{2}(1, t)\right|$ with $\alpha=20, \beta=10$ for example 1 . In Figure 4, we plot absolute error $\left|u(1, t)-u_{3}(1, t)\right|$ with $\alpha=20, \beta=10$ for example 1 . In Figure 5 , we plot absolute error $\left|u(1, t)-u_{1}(1, t)\right|$ with $\alpha=2, \beta=1$ for example 1. In Figure 6, we plot absolute error $\left|u(1, t)-u_{2}(1, t)\right|$ with $\alpha=2, \beta=1$ for example 1. In Figure 7, we plot absolute error $\left|u(1, t)-u_{0}(1, t)\right|$ with $\alpha=20, \beta=10$ for example 2. In Figure 8, we plot absolute error $\left|u(1, t)-u_{1}(1, t)\right|$ with $\alpha=20, \beta=10$ for example 2. In Figure 9 , we plot absolute error $\left|u(1, t)-u_{2}(1, t)\right|$ with $\alpha=20, \beta=10$ for example 2. In Figure 10, we plot absolute error $\left|u(1, t)-u_{3}(1, t)\right|$ with $\alpha=20, \beta=10$ for example 2. In Figure 11, we plot absolute error $\left|u(1, t)-u_{2}(1, t)\right|$ with $\alpha=2, \beta=1$ for example 2. In Figure 12, we plot absolute error $\left|u(1, t)-u_{3}(1, t)\right|$ with $\alpha=2, \beta=1$ for example 2. In Figure 13, we plot exact and numerical solutions with $\beta=1, \alpha=2, n=0$ for example 2. In Figure 14, we plot exact and numerical solutions with $\beta=1, \alpha=2, n=3$ for example 2 .

\section{Conclusions}

In this paper, the main objective of this study was for finding the numerical solution of one dimensional linear hyperbolic telegraph equation, describing wave propagation of electric signals in a cable transmission line. In order to achieve this, we adopted a combination of Laplace transform scheme and homotopy perturbation technique so called Laplace homotopy perturbation method (LHPM). Two typical examples have been demonstrated in order to illustrate the efficiency and accuracy of the present method. It is evident from the solution process that the present method provides a good approximate solution in comparison with the exact solution. Also comparison made with the results obtained by Lakestani and Nemati Saray [8] and Javidi [31]. As can be seen from tables, the present method, considerably more accurate than the other methods. This paper can be used as a standard paradigm for other applications. 


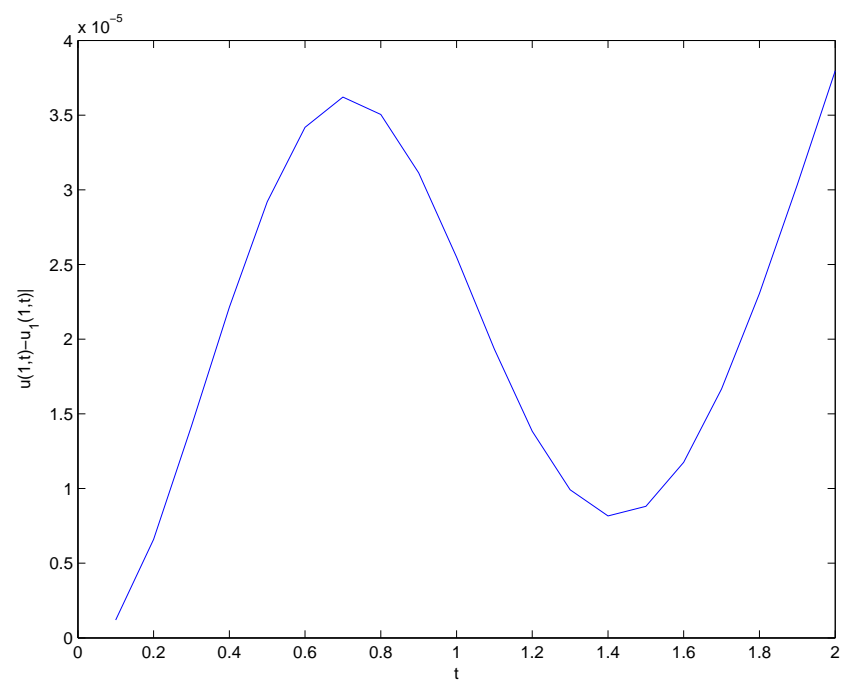

Figure 2: Absolute error $\left|u(1, t)-u_{1}(1, t)\right|$ for $\alpha=20, \beta=10$ for example 1 .

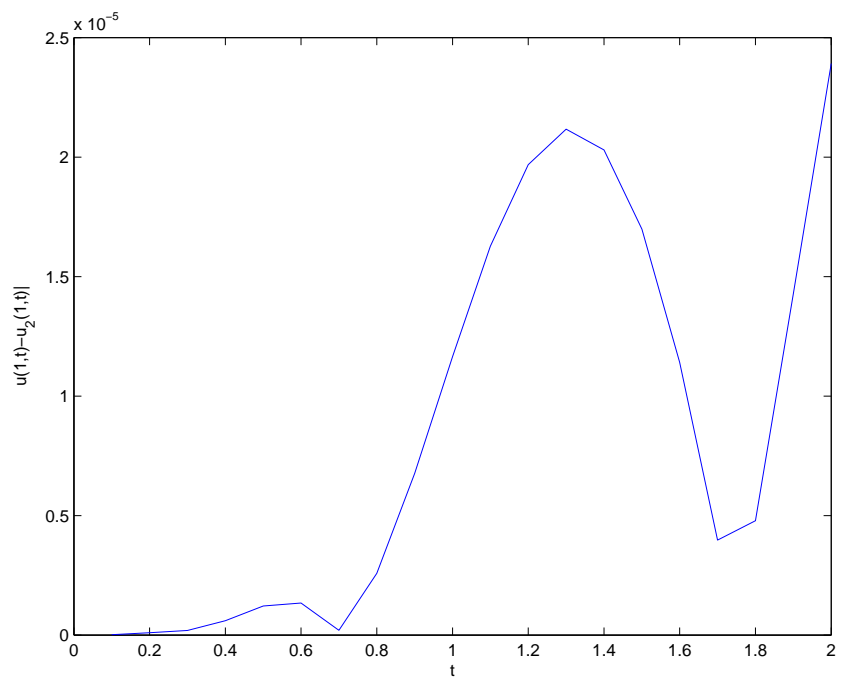

Figure 3: Absolute error $\left|u(1, t)-u_{2}(1, t)\right|$ for $\alpha=20, \beta=10$ for example 1 . 


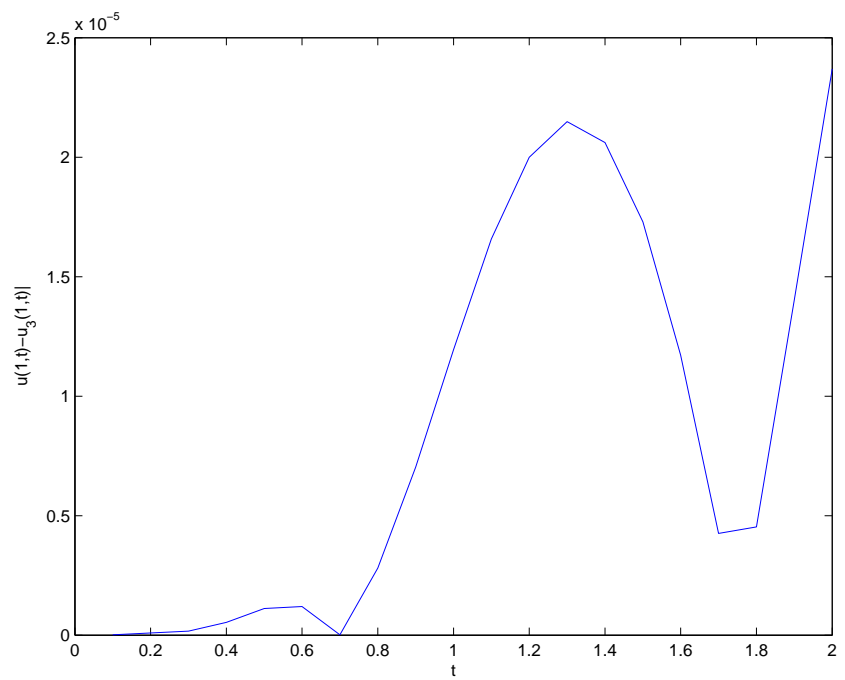

Figure 4: Absolute error $\left|u(1, t)-u_{3}(1, t)\right|$ for $\alpha=20, \beta=10$ for example 1 .

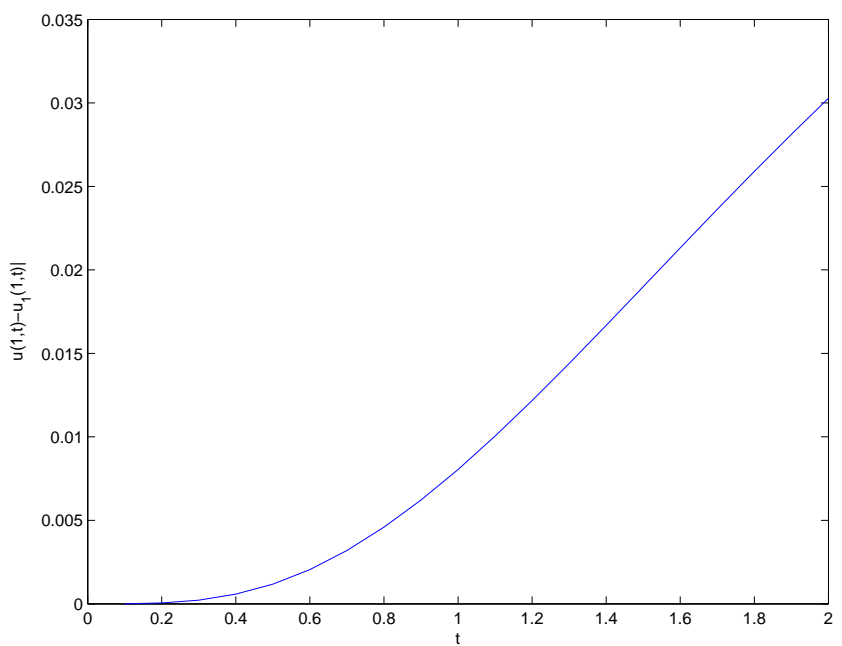

Figure 5: Absolute error $\left|u(1, t)-u_{1}(1, t)\right|$ for $\alpha=2, \beta=1$ for example 1 . 


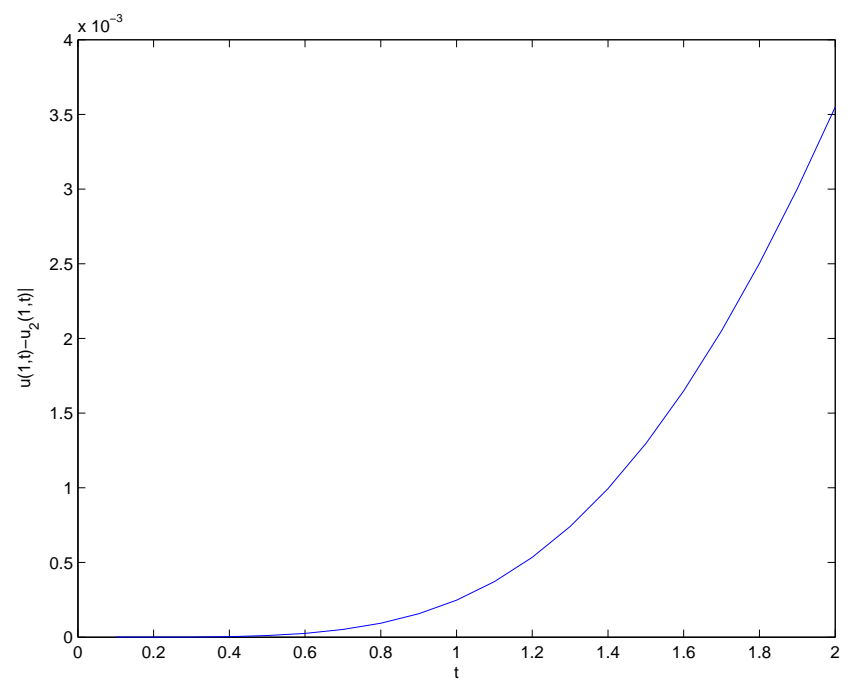

Figure 6: Absolute error $\left|u(1, t)-u_{2}(1, t)\right|$ for $\alpha=2, \beta=1$ for example 1 .

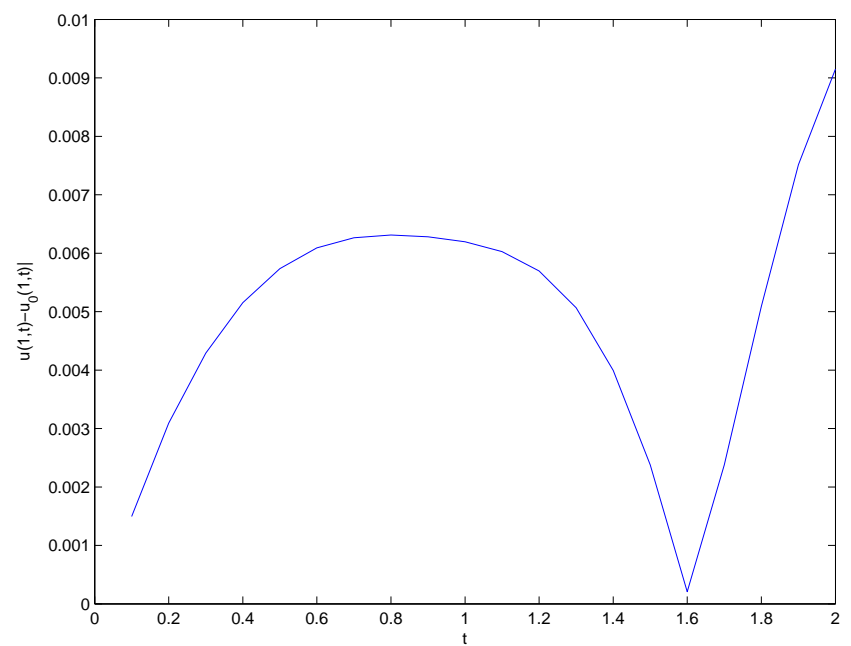

Figure 7: Absolute error $\left|u(1, t)-u_{0}(1, t)\right|$ for $\alpha=20, \beta=10$ for example 2. 


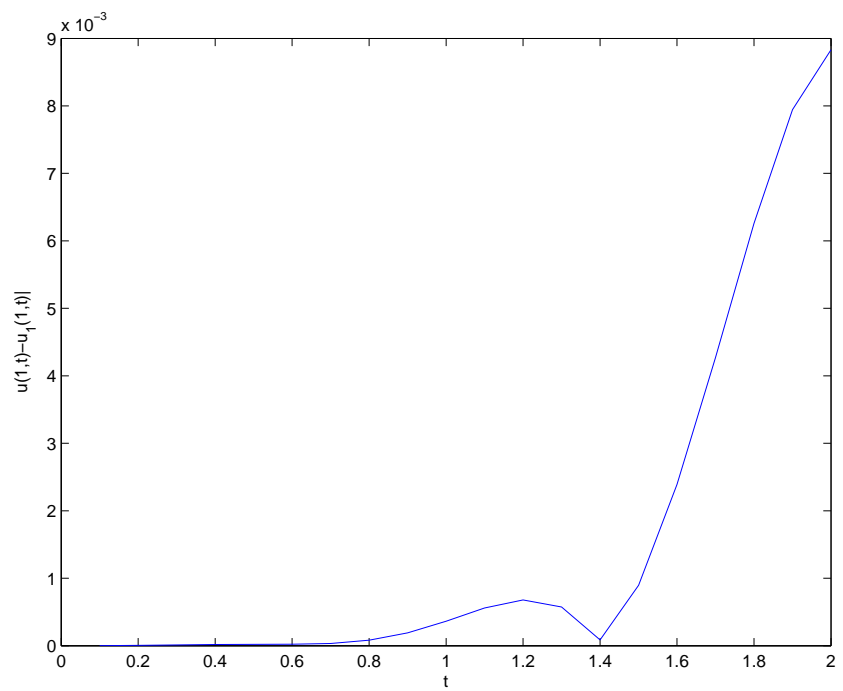

Figure 8: Absolute error $\left|u(1, t)-u_{1}(1, t)\right|$ for $\alpha=20, \beta=10$ for example 2 .

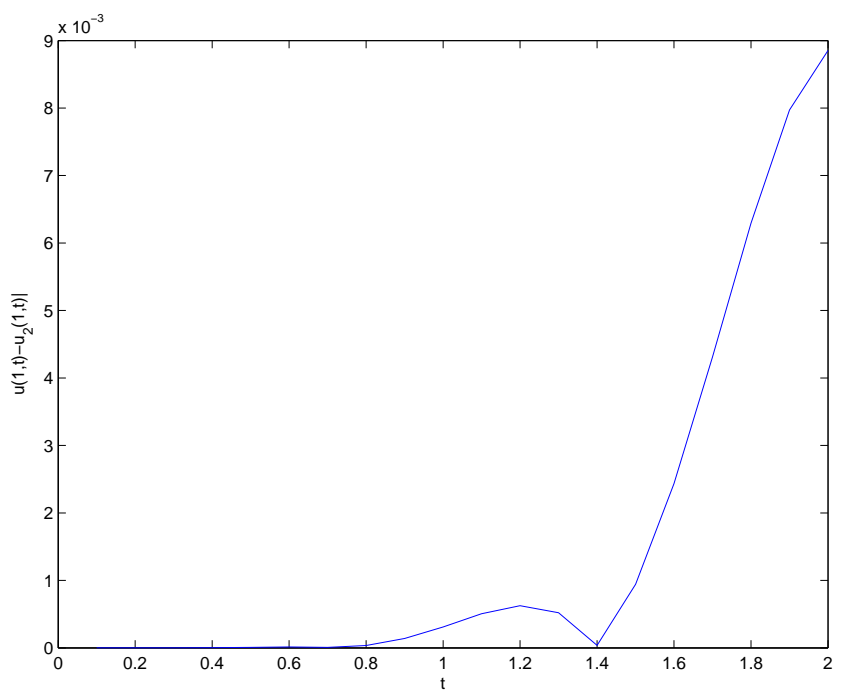

Figure 9: Absolute error $\left|u(1, t)-u_{2}(1, t)\right|$ for $\alpha=20, \beta=10$ for example 2. 


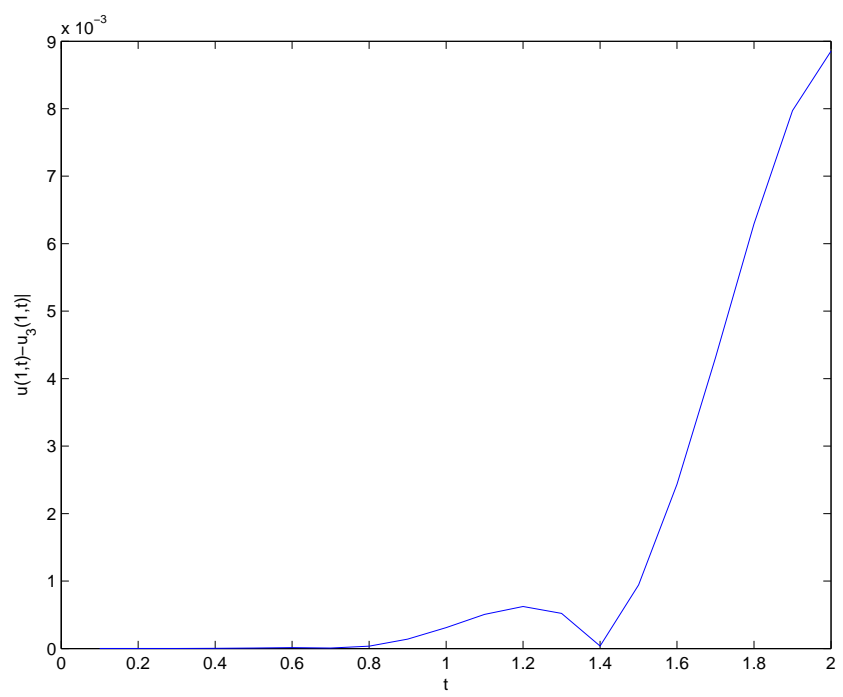

Figure 10: Absolute error $\left|u(1, t)-u_{3}(1, t)\right|$ for $\alpha=20, \beta=10$ for example 2 .

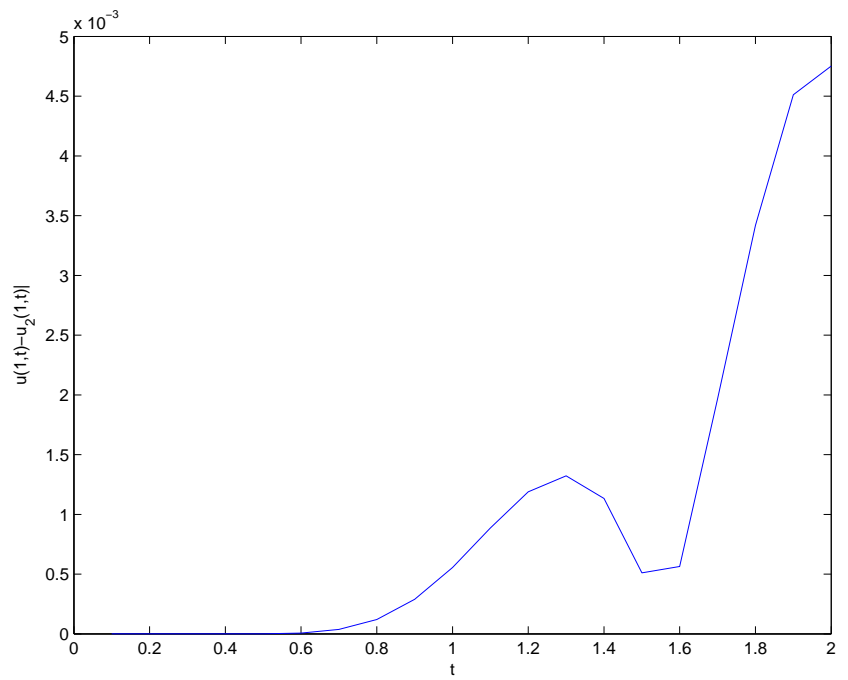

Figure 11: Absolute error $\left|u(1, t)-u_{2}(1, t)\right|$ for $\alpha=2, \beta=1$ for example 2 . 


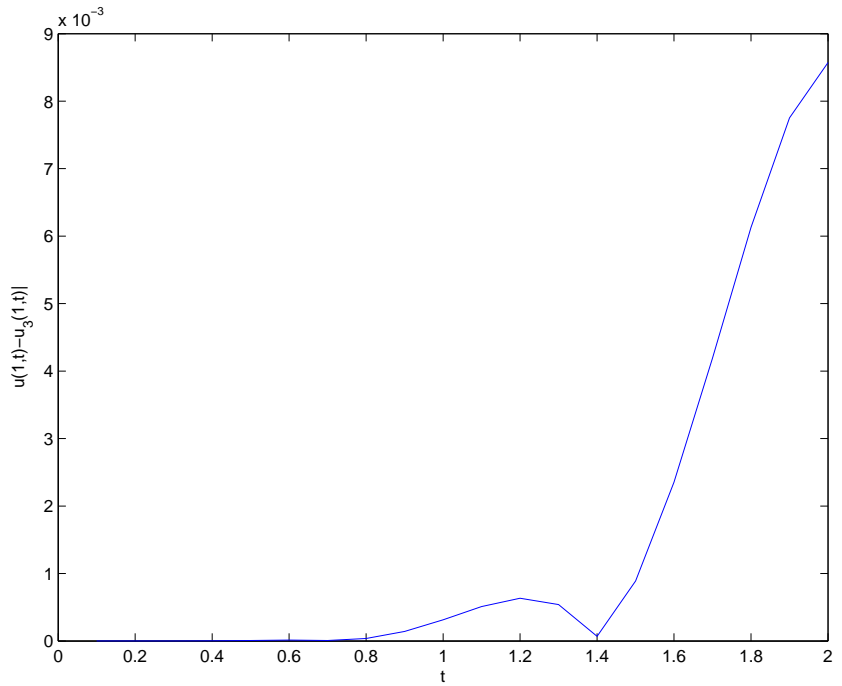

Figure 12: Absolute error $\left|u(1, t)-u_{3}(1, t)\right|$ for $\alpha=2, \beta=1$ for example 2 .

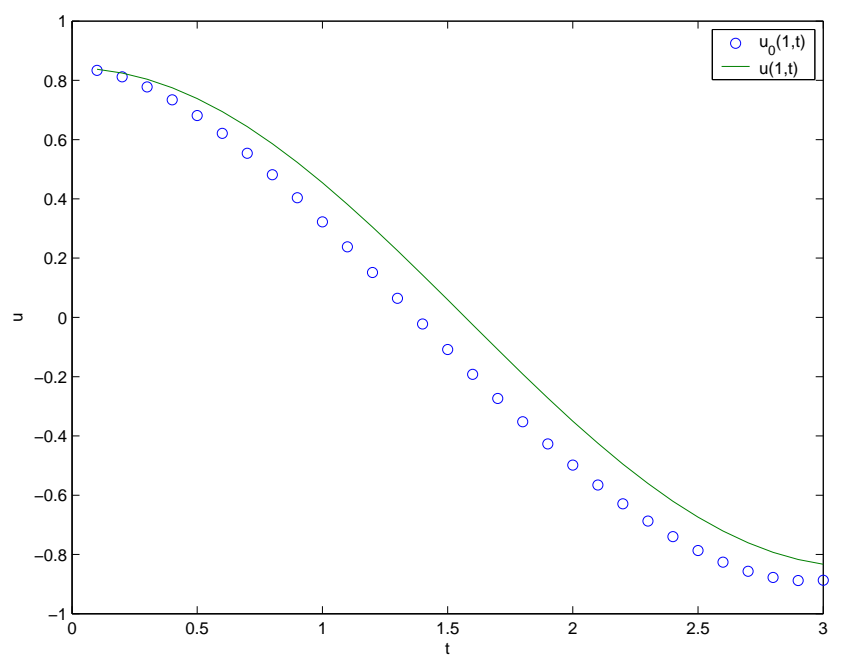

Figure 13: Exact and numerical solutions with $\beta=1, \alpha=2, n=0$ for example 2. 


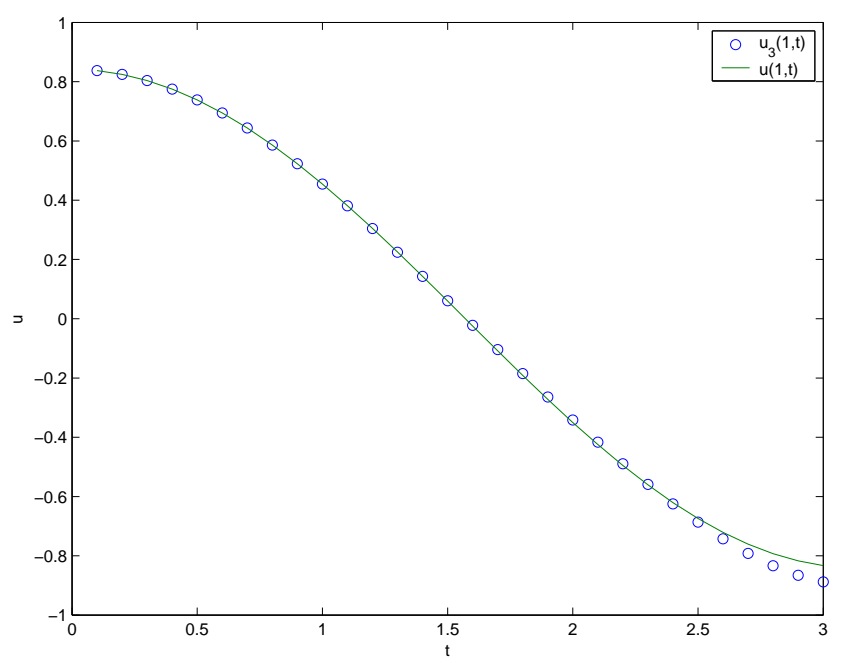

Figure 14: Exact and numerical solutions with $\beta=1, \alpha=2, n=3$ for example 2.

\section{References}

[1] A. Mohebbi and M. Dehaghan, High order compact solution of the one dimensional linear hyperbolic equation, Numerical method for partial differential equations, 24 (2008), pp. 1122-1135.

[2] F. Gao and C. Chi, Unconditionally stable difference scheme for a one-space dimensional linear hyperbolic equation, Applied Mathematics and Computation, 187 (2007), pp. 1272-1276.

[3] A. Saadatmandi and M. Dehghan, Numerical solution of hyperbolic telegraph equation using the Chebyshev Tau method, Numer. Methods Partial Differential Equations 26 (1), (2010), pp. 239-252.

[4] S.A. Yousefi, Legendre multi wavelet Galerkin method for solving the hyperbolic telegraph equation, Numerical Method for Partial Differential Equations, 26(3), (2010), pp. 535-543..

[5] M. Dehghan, A. Ghesmati, Solution of the second-order one-dimensional hyperbolic telegraph equation by using the dual reciprocity boundary integral equation (DRBIE) method, Engineering Analysis with Boundary Elements 34, (2010), pp. 51-59.

[6] S. Das and P.K. Gupta, Homotopy analysis method for solving fractional hyperbolic partial differential equations, International Journal of Computer Mathematics 88, (2011), pp. 578-588.

[7] M.A. Abdou, Adomian decomposition method for solving the telegraph equation in charged particle transport, J. Quant. Spectrosc. Radiat. Transfer 95, (2005), pp. 407-414.

[8] M. Lakestani and B.N. Saray, Numerical solution of telegraph equation using interpolating scaling functions, Computers Mathematics with Applications, 60, (2010), pp. 1964-1972.

[9] R.K. Mohanty, An unconditionally stable difference scheme for the one-space dimensional linear hyperbolic equation, Appl. Math. Lett. 17, (2004), pp. 101-105.

[10] R.K. Mohanty, An unconditionally stable finite difference formula for a linear second order one space dimensional hyperbolic equation with variable coefficients, Appl. Math. Comput. 165, (2005) pp. 229-236.

[11] L. Lapidus and G.F. Pinder, Numerical Solution of Partial Differential Equations in Science and Engineering, Wiley, New York (1982).

[12] I. Hashim, O. Abdulaziz, S. Momani, Homotopy analysis method for fractional IVPs, Communications in Nonlinear Science and Numerical Simulation 14, pp. (2009), 674-684.

[13] A. Borhanifar and R. Abazari, An unconditionally stable parallel difference scheme for telegraph equation scheme for telegraph equation, Math. Probl. Eng., 2009 (2009), Article ID 969610, 17 pages doi:10.1155/2009/969610.

[14] M. Dehghan and A. Shokri, A numerical method for solving the hyperbolic telegraph equation, Numer. Methods Partial Differential Equations 24, (2008) pp. 1080-1093. 
[15] M. Dehghan and M. Lakestani, The use of Chebyshev cardinal functions for solution of the second-order one-dimensional telegraph equation, Numer. Methods Partial Differential Equations 25, (2009), pp. 931-938.

[16] J. Biazar and M. Eslami, Analytic solution for Telegraph equation by differential transform method,Physics Letters A, 374(29), (2010) pp. 2904-2906.

[17] L. Xu, Application of He's parameter-expansion to an oscillation of a mass attached to a streched elastic wire, Phys. Lett. A, 368, pp. (2007), 259-262

[18] J.H. He, Variational approach for nonlinear oscillators, Chaos, Solitons \& Fractals, 34, (2007), pp. 1430-1439

[19] M. Javidi and A. Golbabai, Exact and numerical solitary wave solutions of generalized Zakharov equation by the variational iteration method, Chaos, Solitons \& Fractals,36( 2), ( 2008), pp. 309-313

[20] J.H. He and M.A. Abdou, New periodic solutions for nonlinear evolution equations using Exp-function method,Chaos, Solitons \& Fractals,34(5), (2007), pp. 1421-1429.

[21] A. Golbabai and M. Javidi, A spectral domain decomposition approach for the generalized Burgers-Fisher equation, Chaos, Solitons \& Fractals,In Press, Chaos, Solitons and Fractals, 39 , ( 2009), pp. 385392

[22] G.E. Draganescu and V. Capalnasan, Nonlinear relaxation phenomena in polycrystalline solids. Int J Nonlinear Sci Numer Simulat, 4 (3), (2003), pp. 219-225

[23] J.H. He, A coupling method of a homotopy technique and a perturbation technique for non-linear problems , Int. J. Non-Linear Mech. 35 (1), (2000), pp. 37-43.

[24] J.H. He, The homotopy perturbation method for non-linear oscillators with discontinuities. Appl. Math. Comput. 151(1), (2004), pp. 287-292.

[25] A. Golbabai and M. Javidi, Application of He's homotopy perturbation method for nth-order integro-differential equations, Applied Mathematics and Computation, 190( 2), (2007), pp. 1409-1416

[26] Asymptotology by homotopy perturbation method. Appl. Math. Comput. 156(3), (2004), pp. 591-596.

[27] J.H. He, Homotopy perturbation method for solving boundary problems. Phy Lett. A. 350(1-2), (2006), pp. 87-88.

[28] J.H. He, Limit cycle and bifurcation of nonlinear problems. Chaos, Solitons \& Fractals 26(3),(2005), pp. 827-33.

[29] M. Madani, M. Fathizadeh, Y. Khan, A. Yildrim,, On coupling the homotpy perturbation method ans Laplace transformation, Mathematical and Computer Modelling, 53, (2011), pp. 1937-1945.

[30] H. Stehfest, Numerical inversion of Laplace transform, Comm. ACM 13, (1970), pp. 47-49(see also p. 624).

[31] M. Javidi, Chebyshev spectral collocation method for computing numerical solution of telegraph equation, Compu. Math. with Appl., (2012) Submitted. 